\title{
Optical and Confinement Properties of Two-Dimensional Photonic Crystals
}

\author{
H. Benisty, C. Weisbuch, D. Labilloy, M. Rattier, C. J. M. Smith, T. F. Krauss, \\ Richard M. De La Rue, Senior Member, IEEE, R. Houdré, U. Oesterle, C. Jouanin, and D. Cassagne
}

\begin{abstract}
We describe experiments on a quasi-two-dimensional (2-D) optical system consisting of a triangular array of air cylinders etched through a laser-like $\mathrm{Ga}(\mathrm{Al}) \mathrm{As}$ waveguiding heterostructure. Such a configuration is shown to yield results very well approximated by the infinite 2-D photonic crystal (PC). We first present a set of measurements of the optical properties (transmission, reflection, and diffraction) of slabs of these photonic crystals, including the case of in-plane Fabry-Perot cavities formed between two such crystals. The measurement method makes use of the guided photoluminescence of embedded quantum wells or InAs quantum dots to generate an internal probe beam. Out-of-plane scattering losses are evaluated by various means. In a second part, in-plane micrometer-sized photonic boxes bounded by circular trenches or by two-dimensional photonic crystal are probed by exciting spontaneous emission inside them. The high quality factors observed in such photon boxes demonstrate the excellent photon confinement attainable in these systems and allow to access the detail of the modal structure. Last, some perspectives for applications are offered.
\end{abstract}

Index Terms - Cavity resonators, electromagnetic scattering by periodic structures, Fabry-Perot resonators, luminescence, microcavities, photonic crystals, radiation losses, semiconductor heterojunctions, waveguides.

\section{INTRODUCTION}

$\mathbf{I}$ N THREE-dimensionally periodic dielectric structures, the existence of photonic transmission bands and forbidden bands [1], [2] has opened new avenues to tailor the lightmatter interaction, and in particular spontaneous emission, but also to design new photonic systems with superior properties for photon confinement. At optical wavelengths, threedimensional (3-D) structures have been recently fabricated such as "woodpiles," stacks of dielectric spheres or "inverse opals," etc. [3]-[9]. They are, however, still difficult to fabricate reproducibly with, e.g., the desired index, thickness, and precise quantitative measurements are scarce.

Two-dimensional (2-D) structures, although they do not provide 3-D light propagation or confinement, are

Manuscript received August 12, 1999. This work was supported in part by the EU under the ESPRIT program SMILED.

H. Benisty, C. Weisbuch, D. Labilloy, and M. Rattier are with the Laboratoire de Physique de la Matière Condensée, Ecole Polytechnique, Palaiseau Cedex 91128 France.

C. J. M. Smith, T. F. Krauss, and R. M. De La Rue are with the Optoelectronics Research Group, University of Glasgow, Glasgow G12 8LT Scotland.

R. Houdré and U. Oesterle are with Institut de Micro et Opto-électronique, Ecole Polytechnique Fédérale de Lausanne, Lausanne CH-1015 Switzerland.

C. Jouanin and D. Cassagne are with Groupe d'Etude des Semiconducteurs, Université Montpellier II CC074, Montpellier Cedex 0534095 France.

Publisher Item Identifier S 0733-8724(99)08822-2. much more amenable to controlled fabrication owing to mature nanofabrication technology. Such 2-D structures could already bring a sizeable part of the advantages expected from three-dimensional structures. As a consequence, two-dimensional photonic crystals (2-D PC's) have been more thoroughly investigated than their three-dimensional counterparts [10]-[17]. The aspect ratio of these structures, however, remains a crucial issue: it should be remembered that submicrometer fabrication technologies impose severe limits on the aspect ratio, on the order of ten, with the notable exception of macroporous silicon [14]. Therefore, 2-D PC's should in principle be subject to similar experimental difficulties as their 3-D counterparts concerning the possibility of making precise quantitative measurements when dealing with finite optical beams and PC structures. However, we can design quasi-2-D structures, analogous to quantum wells for electrons, by using waveguided light propagation [10]. In such a case, the optical beam is perfectly defined and collimated, and its interaction with PC's etched across the waveguide can be seen as providing the frame of a well-defined 2-D-physics experiment [18]-[20]. In addition, the waveguide naturally freezes the third degree of freedom: typically $30-40 \%$ of freespace modes are trapped into a dielectric waveguide in current semiconductor systems. Thus, the combination of a PC-based in-plane cavity and a planar waveguide may provide full 3-D light confinement [21]-[23] for those modes.

Semiconductor systems also offer the opportunity to include an efficient internal light source in the waveguide itself, an essential feature in laser heterostructures grown in the wellknown GaAs- or InP-based systems. This is the approach pursued here, whereby the spontaneous photoluminescence of quantum wells or quantum dots embedded in a waveguide is excited by a focused laser spot to produce an internal quasi-2D light beam, which then probes the 2-D PC's (Fig. 1) [18].

Among the advantages of this technique, two proved crucial in understanding the possible light paths after interaction of guided light with 2-D PC's. The first is the ease and accuracy with which such a quasi-point source can be located with respect to the photonic crystals. The second is the very good source reproducibility obtained on account of the growth quality and uniformity of the luminescent layers. This contrasts with the difficulties encountered when coupling lasers to waveguides [24], namely, nonreproducible coupling and parasitic reflection oscillations due to the coherent probe beam.

In the next section, we show how we exploited this advantageous configuration to quantitatively measure all the 


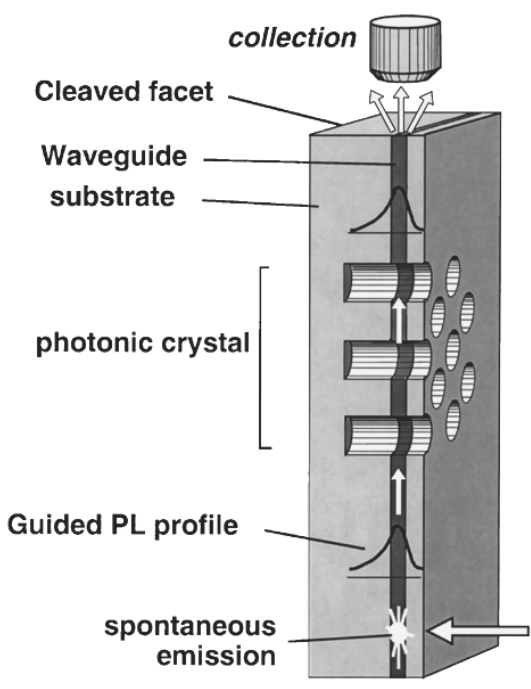

Fig. 1. Principle of the measurement: a two-dimensional photonic crystal is probed by a guided wave, and light is collected at a cleaved facet. Light can be generated by spontaneous emission in the waveguide.

in-plane coefficients describing the interaction of a guided light beam with a photonic crystal [transmission, reflection, and diffraction (TRD)] [19]. The pros and cons of quantum wells versus quantum dot layers as inner sources are also discussed [20]. Next, the main TRD results are summarized, and the significance of some striking features such as high transmission windows and the fine structure of oscillations around the photonic gaps is outlined. In the following section, the amount and the nature of light losses specific to the perforated waveguide are investigated by exploiting the above results (TRD and fine oscillations around gaps) as well as by a new means: the use of one-dimensional (1-D) cavity resonances between two 2-D PC's to measure reflectivities close to unity [22]. This allows us to quantify these losses and to provide a plausible explanation for them. The two last sections are devoted to three-dimensional cavities bounded by photonic microstructures, the modes of which are probed through their internal photoluminescence. In the first case, a concentric Bragg grating confines a new set of microdisc modes, the "quasi-radial modes" (QRM's) [25]. In the other case, the 2-D PC's exemplified above are used to define micrometer-sized hexagonal cavities in the waveguide plane [23]. To support the experimental results, a 2-D supercell calculation of the eigenmodes of a selected cavity is shown. In our conclusion, we evaluate the degree of generality of the results presented above and give some perspectives.

It should be emphasized at this point that the measurement scheme developed here, using a waveguided light beam to study PC properties, is actually in itself a demonstration of the various uses of 2-D PC's in the important fields of spontaneous emission control and integrated optics.

These possibilities include not only the much-quoted example of short bends in a waveguide [Fig. 2(a)] [26], [27]. One can think of cavity modes as filters that drop a selected frequency [Fig. 2(b)] [28], [29]. Diffraction could find its use in wavelength-routing applications [Fig. 2(c)], allowing the implementation of compact spectrometers with low losses.

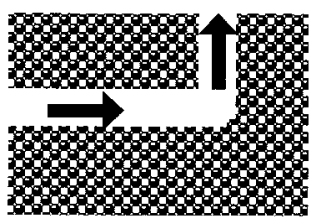

(a)

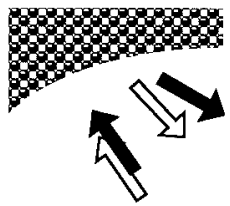

(c)

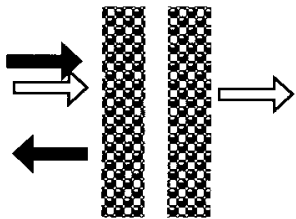

(b)

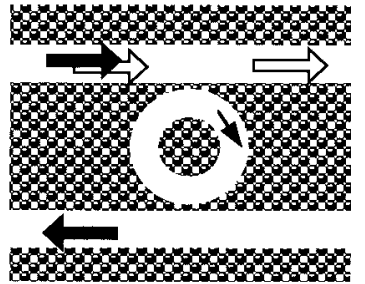

(d)
Fig. 2. Example of possible applications of two-dimensional photonic crystals: (a) short bends in waveguides, (b) filters based on cavity modes select only the resonant frequency, (c) diffraction of a photonic crystal disperses different wavelengths impinging in the same direction, and (d) add/drop filter can be based on waveguides coupled with one or two resonant structures.

Polarization properties may equally affect the design of some integrated optics functions. Of course, in the present era, witnessing the rise of the wavelength domain multiplexing scheme in optical fiber telecommunications, the add-drop filter functionality schematized in Fig. 2(d) is among the most desired [30]. It requires a sophisticated blend of one or two resonant elements and couplers from the basic guides to these elements. The issue of device cascading is one of many that still needs to be addressed carefully and which will be briefly discussed.

\section{Quantitative Measurement of In-Plane INTERACTION OF LIGHT WITH 2-D PC's}

\section{A. Principle}

An optical guided wave traveling in a planar dielectric waveguide is our basic probe beam (Fig. 1). The 2-D PC's that act on such a beam are arrays of cylindrical air holes normal to the guide plane. One initial reason to study such crystals was that they may display a photonic bandgap in both polarizations [denoted transverse electric (TE) and transverse magnetic (TM)] for a triangular lattice and a sufficiently large air-filling factor $f$. However, it became obvious a few years ago that large air holes could be very detrimental to waveguiding and lead to losses out of the guide [10], [24]. This prompted experimentalists to consider only crystals with moderate air-filling factors, on the order of $f=0.30$, in which air hole diameters are well in the subwavelength regime, leading to a drastic reduction of out-of-plane scattering. In these crystals, the dielectric is highly connected, resulting in a full TE gap with reduced frequency $a / \lambda$ around 0.23 [instead of 0.45 around the "optimal" filling factor $f \approx 0.7$ (see Fig. 7)], while directional TM gaps are rather narrow and do not overlap anymore for such low $f$ values.

Next, one should produce and collect optical probe beams. As mentioned above, luminescent layers such as quantum 
wells or self-organized quantum dots located in the waveguide itself are a very convenient source. We used GaAs/AlGaAs heterostructures containing a central monomode GaAs waveguide of $0.24 \mu \mathrm{m}$ thickness, with an asymmetric GaAlAs clad [18]. A red laser diode is used to photoexcite these active regions, and about $30-40 \%$ of the radiative recombination is channeled into the waveguide mode, the rest escaping through the substrate or the air. Direct collection in air just amounts to a standard photoluminescence experiment, although only the guided part is of interest in the following.

If the laser spot is far enough from the PC's under test, the point source approximation is valid and the guided beam has almost straight wavefronts. It is not quite so when photoluminescence is excited close to, or even within, a photonic structure, a situation met in the two last sections. It leads to some additional phenomena (directionality [31] or lifetime [32] changes), which will not be considered in this paper.

For collection, it is convenient to take advantage of a cleaved edge located at a few tens of micrometers of the PC's [18]. As will be shown later, the ability to collect light with a good degree of spatial resolution is essential when assessing properties of the PC's.

An alternative way of collecting guided light could be to use a grating coupler to the air. It turns out that, to a smaller degree, scattering to the air by the PC plays a similar role in some of the crystals or etched mirrors used to define cavities below. Surface emitted light is more easily detected in this latter case because the source is within a micrometer or so of the photonic structures, giving rise to a larger absolute scattered intensity.

\section{B. Setup}

Two optical axes coincide at the sample location: one normal to the sample surface, the other normal to the cleaved edge [Fig. 3(a)] [18]. The first, denoted the front axis [horizontal in Fig. 3(a)], is used to excite photoluminescence and view the sample surface. The PL spot is located at a distance $d$ on the order of $20-200 \mu \mathrm{m}$ from the cleaved edge. We use dichroic mirrors and filters to observe this front surface and collect all the 900-1100-nm infrared beams (PL + visualization) onto an ordinary charge-coupled device (CCD) camera.

The second axis, denoted the lateral axis [vertical in Fig. 3(a)], is at right angles with the former. We use a microscope on this axis, with apertures ranging from 0.25 to 0.4 , and again infrared imaging is performed by means of an ordinary CCD. The sample, mounted on a goniometer head, is carefully aligned with both axis.

Spectral analysis may be performed with a resolution of a few micrometers on the front or lateral axes: In front of each CCD, beam splitters direct the magnified image into a multimode optical fiber, which guides photons toward an optical multichannel analyzer, with a cooled Si-CCD detector (detection limit $\approx 1060 \mathrm{~nm}$ ). This spatially resolved analysis is crucial in selectively collecting guided light rather than other beams [18]. Fortunately, two factors combine to enhance this selectivity.

1) Neglecting any absorption, the nonguided beams decay as $1 / d^{2}$, whereas the guided beams decays as $1 / d$.

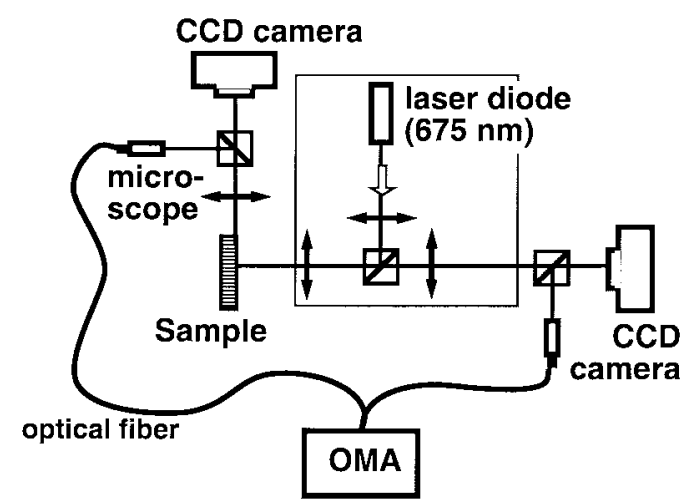

(a)

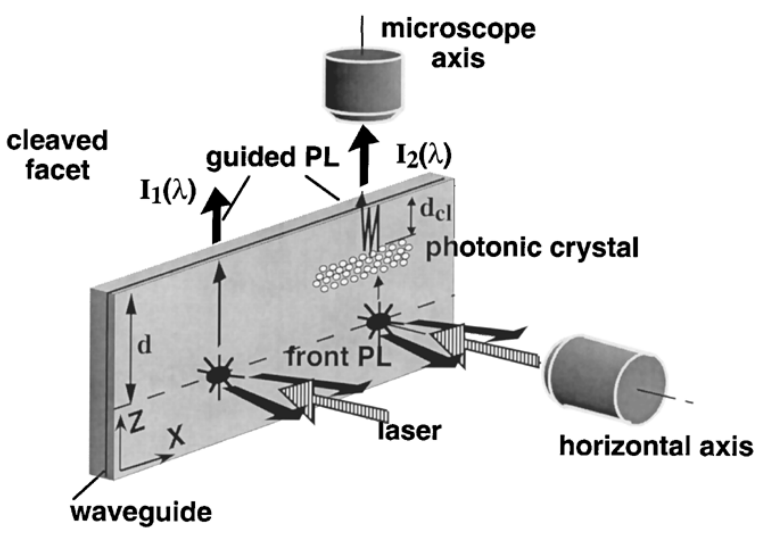

(b)

Fig. 3. (a) The measurement setup: two optical axes coincide at the sample location, one normal to the sample surface, the other normal to the cleaved edge. Both front and side photoluminescence are spatially resolved by the fibers $(100-\mu \mathrm{m}$ core) before being spectrally analyzed by the optical multichannel analyzer (OMA). (b) Configuration used for PC transmission/reflection measurements: excitation is performed at a constant distance $d$ from the edge; the reference is taken in an unetched area. When light traverses the photonic crystal, interferences between the crystal and the cleaved edge arise.

2) The intensities of the former beams rapidly vanish at grazing incidences. Snell's law predicts that the air beam is coupled in this limit to vanishing internal angles; for the substrate beam, it has to "tunnel" through the thick Al-rich clad layer before propagating beneath the guiding structure, a mechanism that also causes an important attenuation.

Finally, refraction and Snell's law also naturally provide us with an internal angular selectivity of a few degrees around the normal to the cleaved edge: the largest angle collected in the microscope is $\sin ^{-1}\left(N A / n_{\mathrm{eff}}\right)$, where $n_{\mathrm{eff}}$ is the effective index of the waveguide mode. We used an aperture $N A=0.4$, resulting for $n_{\mathrm{eff}} \approx 3.35$ in a maximum internal angle of $\theta=6.85^{\circ}$. Since many of the basic photonic features such as the Fabry-Perot resonances, investigated below, have a basic $1 / \cos \theta$ behavior, this angular selectivity translates into a spectral selectivity better than $0.7 \%$ if all the emerging directions are collected. The spatially resolved detection into the fiber also selects a still narrower angular range of $1-2^{\circ}$. For $\theta=2^{\circ}$, the effects discussed above are smaller than the spectrograph resolution $(0.05 \%)$. 
The system is mounted in such a way that the front optical axis can be moved freely below the microscope with perfectly constant excitation conditions. The whole experiment (except the OMA) is very compact. For further studies, cooling of the sample between the two objectives would be the main tour de force that would still enhance our capabilities.

\section{Measurement of Reflection and Transmission}

Fig. 3(b) describes the configuration used for PC transmission measurements [18], [19]: excitation is performed at a constant distance $d$ from the edge. To get a reference signal $I_{1}(\lambda)$, the guided light intensity is measured in a nonprocessed area, separately in both TE and TM polarizations. There are three attenuation mechanisms with respect to a usual plane wave.

1) The probe beam diverges and decays as $1 / d$.

2) There is some reabsorption of the generated guided light between the spot and the cleaved edge, particularly strong in the case of quantum wells at photon energies above the exciton energy, but still in the linear absorption regime, as checked experimentally by varying $d$.

3) The facet transmission.

However, when placing a photonic crystal into the light path, at a distance $d_{\mathrm{cl}}$ from the cleaved edge, and measuring the new intensity $I_{2}(\lambda)$, the two first mechanisms do not change in magnitude. The third mechanism turns out to provide multiple-beam interferences between the photonic crystal and the cleaved edge. Accordingly, fringes appear in the transmitted spectra, the ratio $I_{2}(\lambda) / I_{1}(\lambda)$ being described by the classical Fabry-Perot-type transmission formula

$$
T_{\mathrm{FP}}(\lambda)=T \times\left|\frac{1}{1-r r_{2} \exp \left(4 i \pi d n_{\mathrm{eff}} / \lambda\right) \exp \left(-\alpha d_{\mathrm{cl}}\right)}\right|^{2}
$$

where $r_{2}$ and $r$ are, respectively, the cleaved edge and the PC amplitude reflectivities, and $\alpha$ is the guided mode absorption coefficient. The fringe visibility thus offers a convenient means to measure the crystal's amplitude reflectivity, under the assumption that the wavelength-dependent absorption factor $\alpha d_{\mathrm{cl}}$ is known and moderate. The choice of the distance $d_{\mathrm{cl}}$ (depending on the ability to cleave at a predetermined position) is thus a compromise between absorption (low $d_{\mathrm{cl}}$ ) and an optimal pitch of the fringes with respect to the photonic features such as gap edges, etc., on the one hand and the spectrometer resolution on the other hand. Other factors that affect the fringe visibility are the finite extent of the source and of the collection region, which both introduce variable angles of the probe waves, and the tilt $\gamma$ between the crystal face and the cleaved facet, which causes fringes to be spatially localized within a length on the order of $\lambda / 2 \gamma$.

In the case of quantum wells (QW's), due to reabsorption of the guided mode by quantum-well excitons, the probed wavelength range is usually quite narrow (about $20 \mathrm{~nm}$ wide only), so that only a small part of the band structure can be checked with one slab of crystal. Since the spectral behavior of a (perfect) photonic crystal scales with the dimensionless normalized energy $u=a / \lambda$, instead of varying $\lambda$, we fabricated

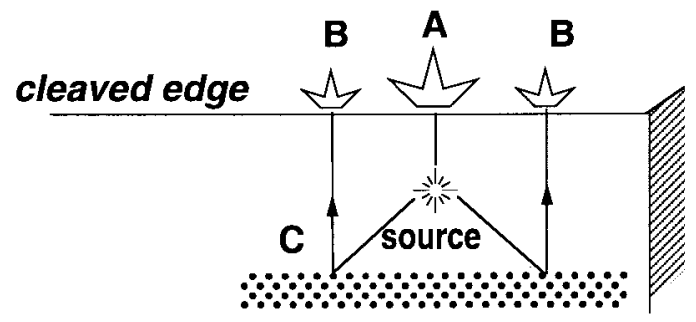

(a)

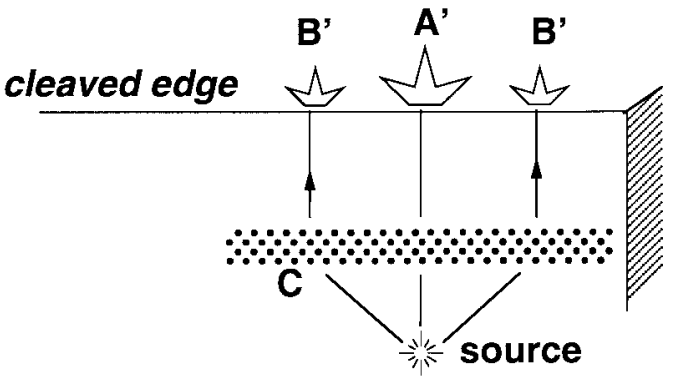

(b)

Fig. 4. (a) Diffraction geometry used to detect backward diffracted beams and (b) same for forward diffracted beams.

photonic lattices with seven different periods at a constant airfilling factor [a technological challenge (see [10])], ranging from $a=180 \mathrm{~nm}$ to $a=360 \mathrm{~nm}$, in order to probe a large range of $u=a / \lambda$ from 0.18 to 0.4 . The different crystal axes were probed by fabricating two types of pattern, with either the $\Gamma M$ or the $\Gamma K$ principal crystallographic axis of the Brillouin zone aligned along the probing beam (i.e., normal to the cleaved edge).

\section{In-Plane Diffraction Measurements}

Photonic crystals exhibit a gap at those frequencies that build up constructive interferences that prevent light propagation. A plane wave at a gap frequency impinging on a crystal, however, has no reason to be specularly reflected: it can also be diffracted. It is not a surprise that a periodic object gives rise to diffraction, all the more so if the impinging beam travels in a media of higher index than the average photonic crystal index. These diffraction effects have been assessed theoretically [33]. Experimentally, we use the geometry of Fig. 4(a) to detect possible diffracted beams in the reflection geometry, and that of Fig. 4(b) in the transmission geometry [19]. In the case of quantum wells, a narrow wavelength range is present in the measurement, the Bragg angle for diffraction is almost uniquely defined, and two diffracted beams appear on the CCD in the form of secondary virtual sources beneath the crystals. The proper way to take into account the geometry of the measurement to extract a meaningful diffraction efficiency figure is described in [19] and [34].

\section{E. Quantum Wells Versus Quantum Dot Luminescent Layers}

We discussed above the fact that due to the reabsorption by excitons, quantum-well layers caused a strong reabsorption for most of the photoluminescence spectrum generated into the waveguide modes, allowing large propagation lengths (tens 


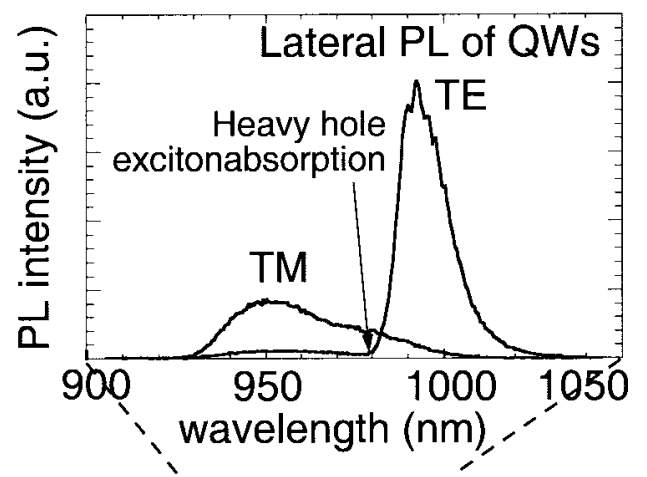

(a)

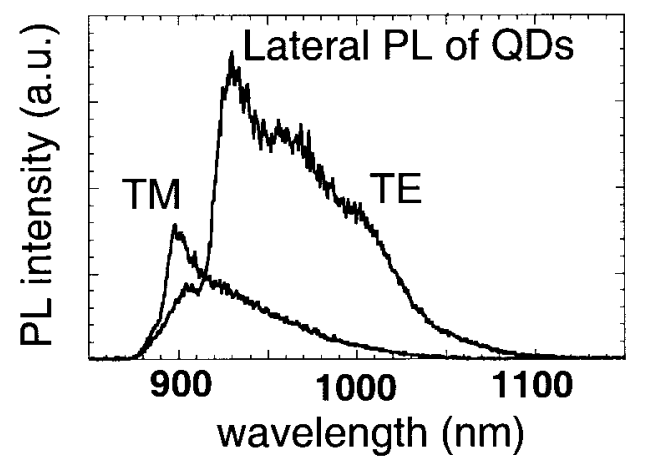

(b)

Fig. 5. (a) Lateral photoluminescence for a heterostructure containing three quantum wells, showing for TE polarization the strong heavy hole absorption edge at $980 \mathrm{~nm}$ that restricts the useful range to about $20 \mathrm{~nm}$. The companion light-hole absorption edge lies at $940 \mathrm{~nm}$. (b) Lateral photoluminescence for a heterostructure containing three layers of self-organized InAs quantum dots, at a few tens of micrometers from the edge. After a larger distance, the relative intensity of the short wavelength side decays, and the maximum shifts toward $1000 \mathrm{~nm}$ for the TE polarization.

of micrometers or more) only in the low-energy tail of the spectrum. This is visible in Fig. 5(a), in which the sharp edge stemming from excitons at $980 \mathrm{~nm}$ is clearly visible on the guided TE spectrum, together with the slight recovery of the signal at smaller wavelengths. The other spectrum represents a typical TM spectrum with slightly less marked features. Let us recall that electron-heavy-holes (respectively, light holes) recombinations are rather TE (respectively, TM) polarized and occur at different energies, but that in the present case, due to the $1-2 \%$ compressive strain in the quantum well, the polarization selection rules are still more apparent.

The narrow spectra and short reabsorption length both can be overcome by the use of luminescent self-organized InAs quantum dot (QD) layers. When growth is performed in such a regime that a deliberately broad size distribution of the dot occurs, it translates into an inhomogeneously broadened spontaneous emission spectrum, with widths of several hundreds of nanometers. Absorption is also spectrally diluted, so that the absorption coefficient is much less than 100 $\mathrm{cm}^{-1}$ in a broadband at wavelengths from about $940 \mathrm{~nm}$ to at least above our detection threshold of $1060 \mathrm{~nm}$ [see Fig. 5(b)]. The inconvenience of this broadened spectra is the smaller intensity available per unit bandwidth (or equivalently per spectrometer pixel) and the slightly poorer photoluminescence yield homogeneity due to the random nature of the growth.

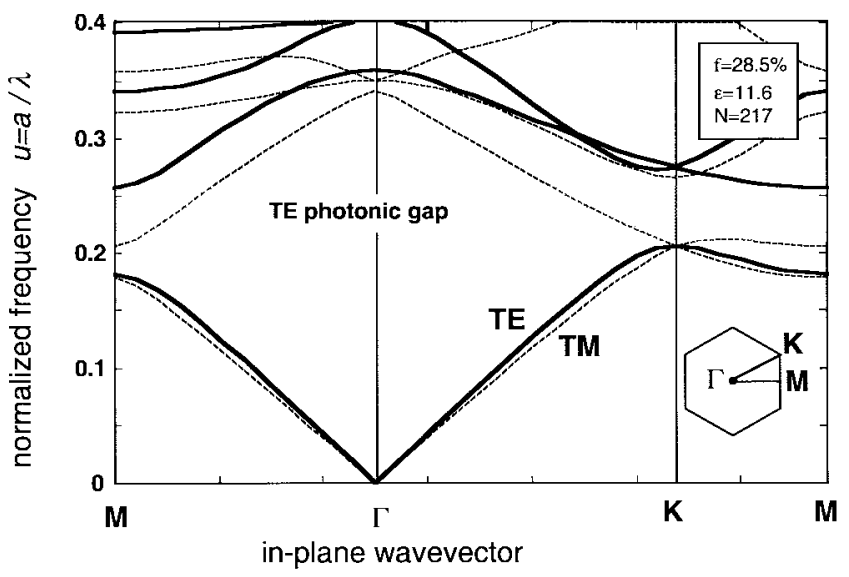

Fig. 6. Band structure of a 2-D PC with an air-filling factor $f=0.285$ and dielectric constant $\varepsilon=11.6$, calculated with 217 plane waves. We use $a / \lambda$ as the normalized frequency. The first Brillouin zone is sketched in the inset.

However, with three InAs layers, a fairly good uniformity was obtained, typically below $\pm 10 \%$ at the scale of the patterns $(100 \mu \mathrm{m})$. For better accuracy, we used the wavelengthintegrated front PL of QD's to compensate this fluctuation.

An important advantage of QD's is their better immunity to nonradiative recombination at etched interfaces, due to the fact that carrier are trapped in the dots. This trapping is far from perfect at room temperature, carriers still "evaporate" from the dots, and some diffusion to the etched interfaces is still likely to occur, but to a much reduced degree compared to quantum wells. On the other hand, when considering structures for device applications, QW's might retain some advantages: for light-emitting-diodes, for example, the higher density-ofstates of QW's can lead to higher injection levels before reaching saturation, which translates into higher brightnesses (radiances).

\section{OPTICAL PROPERTIES OF 2-D PC's: REFLECTION, TRANSMISSION, AND DIFFRACTION}

\section{A. Basic Predictions for 2-D Photonic Crystals}

Fig. 6 gives an example of the band structure of a triangular 2-D photonic crystal made of cylindrical holes with filling factor $f=0.285$ in a dielectric matrix $\varepsilon=11.6$, calculated by the plane wave method [35]. We use again the normalized frequency $u=a / \lambda$. A full gap forms only for the TE polarization, between $u=0.21$ and $u=0.255$. For a $1-\mu \mathrm{m}$ probe wavelength in vacuum, the corresponding crystal periods range from $a=210 \mathrm{~nm}$ to $a=255 \mathrm{~nm}$. Due to the smaller $k$ at the Brillouin zone edge, the $\Gamma M$ stopband is centered at a lower frequency than the $\Gamma K$ stopband but has a similar width. In the TM polarization, a stopband at frequencies just below those of the TE stopband can be predicted along $\Gamma M$. Note that such a diagram does not predict all the transmission gaps: a stopband can also arise due to the absence of coupling between an incident plane wave and the $k$-matched propagating Bloch mode [36]-[38].

Since the air-filling factor $f$ may vary owing to the fabrication process, the "gap map" of Fig. 7 depicting the gaps 


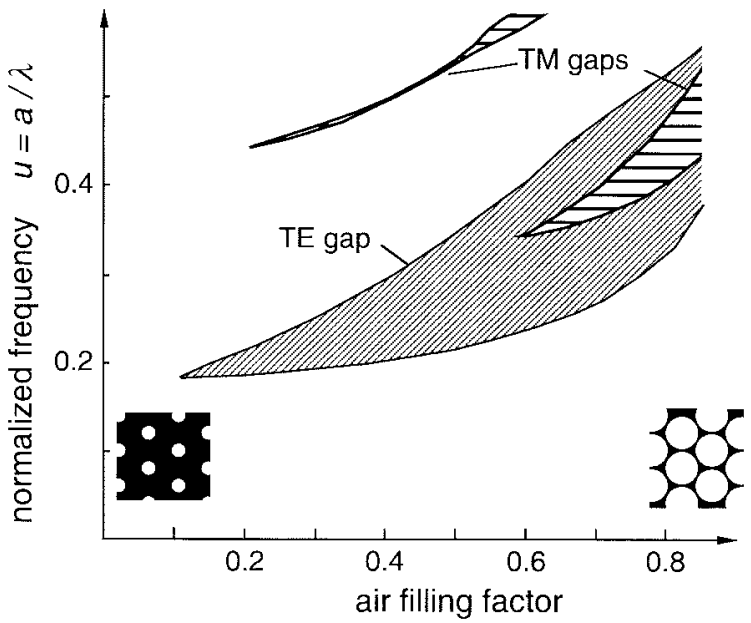

Fig. 7. Map of photonic bandgaps in both polarizations for a triangular lattice of cylindrical holes in a dielectric matrix $\varepsilon=13.6$ in the $(f, a / \lambda)$ plane.

as a function of $f$ is an important piece of information (it is done here for GaAs with $\varepsilon=13.6$ : this lowers the frequencies and slightly broadens the gaps with respect to $\varepsilon=11.6$ ). It is seen that the TE gap (favored by connected dielectric structures) forms around $f=0.10$ and increases linearly beyond. The frequency of the lower TE band edge, the "dielectric band" (because the mode at this band edge is localized in the dielectric; it is also called the "valence" band [2]), is roughly independent of $f$ in this low- $f$ region as the fields in these modes tend to overlap air holes as little as possible. The opposite behavior holds for the upper band edge ("air" or "conduction" band), growing rapidly with $f$, because the field of these modes is more present in air holes. The TE gap width is thus a good measure of the air-filling factor. Note also that filling factors of about 0.6 are needed to obtain overlapping TE and TM gaps.

Comparing our data to these purely two-dimensional predictions, as done below, is a test assessing whether the waveguide configuration, a quasi-2-D system, can represent a 2-D physics experiment on the same footing as an infinitely extended array of cylinders probed by a normal plane wave.

\section{B. Quantum-Well Layers: Reflection and Transmission}

All subsequent data in this section are taken through 15-unit-cells-thick quasi-infinite slabs of photonic crystal, $30 \mu \mathrm{m}$ long. Measured transmission $T(u)$ (points) and their derivatives $\partial T(u) / \partial u$ (arrows) are reported for all seven samples and the two directions of propagation $\Gamma M$ and $\Gamma K$ [19]. Lines are guides to the eye. Data are shown first for the TE case where a gap is expected [Fig. 8(a)]. For comparison, we show [Fig. 8(b)] the theoretically predicted transmission (using transfer matrix method) of a triangular array of infinitely deep air cylinders in a uniform dielectric, below the experimental data. Parameters used in the calculation were $f=28.5 \%$, consistent with experimental values, and dielectric constant $\varepsilon=10.2$, somewhat lower than the effective index of the waveguide.

The general behavior of intensities as well as derivatives is very consistent with the calculation. In particular, one can (a)

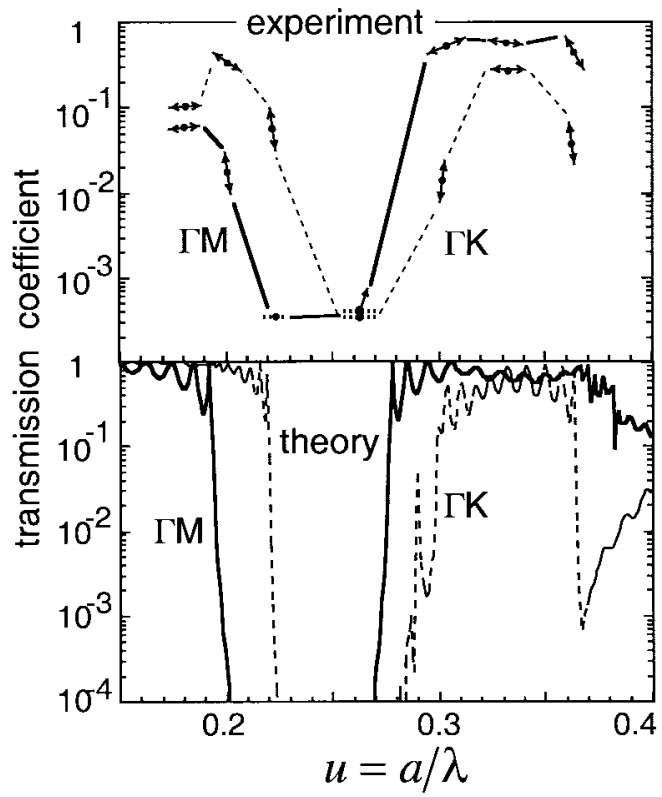

(c)

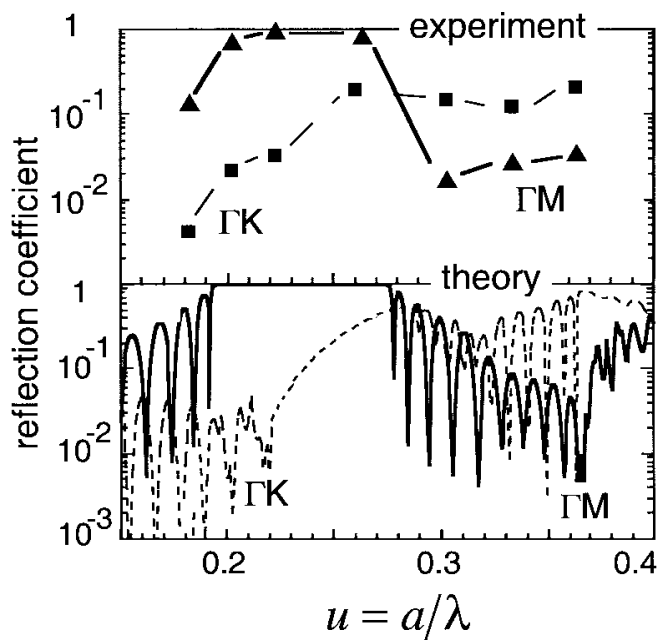

Fig. 8. Transmission and reflection from a 15-row crystal. (a) TE experimental points and derivatives for $\Gamma M$ (solid lines) and $\Gamma K$ (dashed lines) orientations. The lines are guides to the eye only. (b) Corresponding theoretical transmission of a purely two-dimensional PC of 15 rows for $\varepsilon=10.2$ and a filling factor $28.5 \%$. (c) Experimental reflection data for $\Gamma M$ (bold lines) and $\Gamma K$ (thin lines) orientations. (d) Theoretical reflection of a purely two-dimensional PC of 15 rows for $\Gamma M$ (solid lines) and $\Gamma K$ (dashed lines) orientations.

note the relative positions of $\Gamma M$ and $\Gamma K$ curves and the two overlapping stopbands around $u=0.25$, going down to the noise level. Clear falling band-edges appear at $u=0.2$ for $\Gamma M$ and $u=0.23$ for $\Gamma K$. In the pass window between $u=0.3$ and $u=0.35$, transmission in excess of $50 \%$ is observed not far from the theoretical value. The contrast between pass and stopbands exceeds three orders of magnitude.

A crucial test showing that in-plane interference effects dominate the photonic crystal behavior (as opposed to outof-plane scattering that would diminish transmission as well) is that low transmission regions display high reflection as well. Reflection data obtained from the analysis of fringe visibility are shown in the bottom frames [Fig. 8(c)]. Unlike transmission data, only points are displayed since a derivative cannot be deduced with the small number of fringes spanning 
(a)

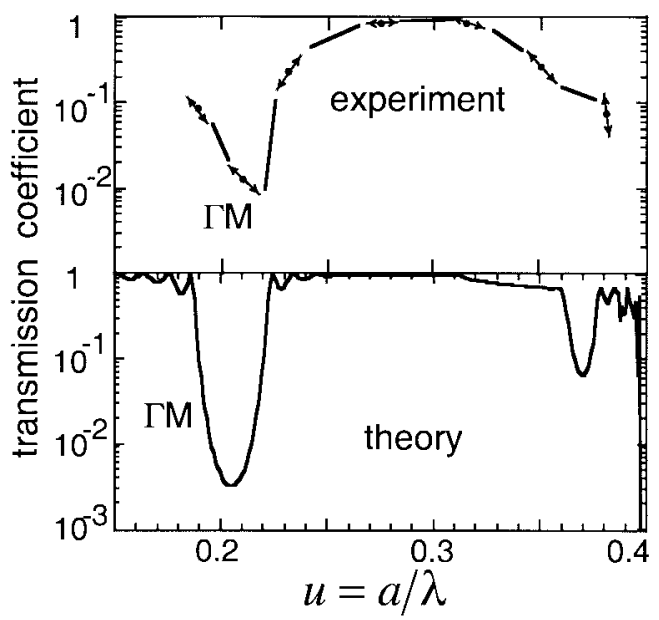

Fig. 9. (a) Same as Fig. 8(a) for TM polarization and $\Gamma M$ orientation. (b) Corresponding theoretical transmission of a purely two-dimensional PC of 15 rows; note the plateau around $u=0.28$.

the 20-nm spectral window. Again, a very satisfying agreement with theory is found [Fig. 8(d)]. The highest reflectivity of $R>80 \%$ is obtained for TE polarization propagating along $\Gamma M$, coinciding with the low transmission window.

Fig. 9(a) shows transmission data for TM polarization along the $\Gamma M$ direction. It is especially striking because of the very high transmission obtained around $u=0.30$, a region where theory predicts a particularly flat pass-window, very close to unity [Fig. 9(b)]. This is an interesting situation as light traverses 30 dioptric interfaces and still retains most of its intensity. This can be viewed as a nice demonstration of the existence of propagative Bloch modes even in a strongly variable dielectric landscape, in some respects at least as impressive as the existence of strong stopbands.

\section{In-Plane Diffraction}

Although in-plane diffraction of a wave impinging on a 2-D photonic crystal at a gap frequency has been predicted [33], its experimental demonstration partly came as a surprise, as it was often thought that a full photonic bandgap would provide a perfect mirror, implicitly meaning a mirror with $100 \%$ specular reflection.

In-plane diffraction effects are basically determined by the full 2-D periodicity of the PC. One limit case is kinematic "volume" diffraction as is usual for X-rays, and for which the reciprocal lattice has to coincide exactly with the Ewald sphere. But due to large dynamical effects (e.g., attenuation of the incident wave on the successive rows), the details of which are beyond the scope of this paper, conditions for diffraction are much relaxed from exact coincidence. It can then be shown that the diffraction cutoff rules for guided waves impinging onto a PC slab are similar to those of diffraction by a plane grating [19] having the periodicity $a_{s}$ of the slab boundary. For the crystals under test here, $a_{s}=a$ for $\Gamma M$ slabs and $a_{s}=$ $\sqrt{3} a$ for $\Gamma K$ slabs. As a result, diffraction at normal incidence is allowed for normalized frequencies $u \geq\left(n_{\mathrm{eff}}\right)^{-1} \approx 0.29$ for $\Gamma M$ slabs and $u \geq\left(\sqrt{3} n_{\mathrm{eff}}\right)^{-1} \approx 0.17$ for $\Gamma K$ slabs, including the TE gap frequency only in this latter case. Only below these cutoffs should one observe $R+T=1$. Above, four plane (a)

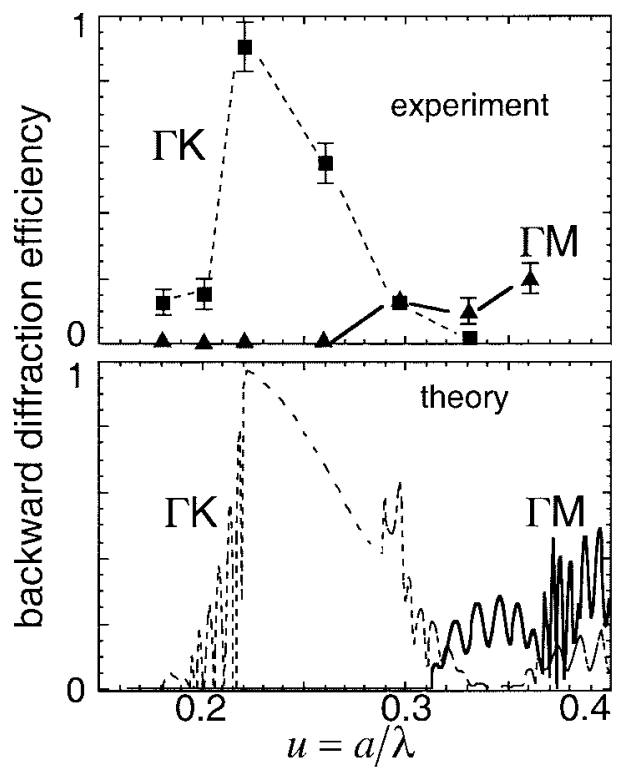

(c)

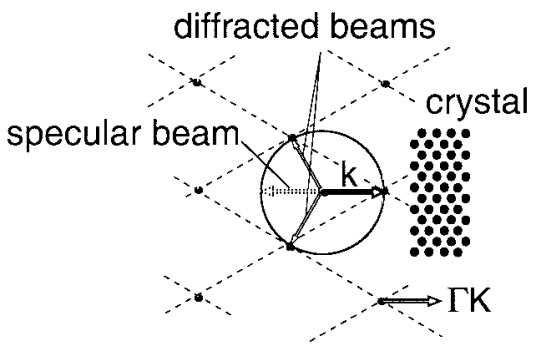

Fig. 10. (a) Experimental measurement at normal emergence of the diffraction efficiency into the two first-order backward beams (taken together) for $\Gamma M$ (solid lines) and $\Gamma K$ (dashed lines) directions normal to the crystal. (b) Theoretical efficiency. Note the cutoff around $u=0.30$, which is too large in the theory because this latter assumes a too-low effective index (3.2 instead of 3.4). (c) 2-D Ewald construction: reciprocal lattice (points and dashed lines) of the crystal for $\Gamma K$ orientation, Ewald circle, normally incident wave (bold arrow), diffracted waves (thin arrows), and specular reflected wave (dashed arrow).

waves are likely to be diffracted in first-Bragg orders at angle $\theta$, two with efficiencies $\eta_{R}$ and two with efficiencies $\eta_{T}$ (see Fig. 4). Lossless interaction of a guided wave with the crystal now reads $R+T+D=1$, where $D=2 \eta_{R}+2 \eta_{T}$ is the diffracted power for unit incident power. As for the absolute magnitude of $\eta_{R}$ and $\eta_{T}$, the relaxed Ewald conditions still tell us that these magnitudes depend on the proximity of the associated reciprocal lattice vector with the Ewald sphere, as illustrated below.

The measured diffraction efficiencies are shown in Fig. 10(a) for TE polarization in the backward diffraction geometry. They are compared to calculated curves for $2 \eta_{R}$, using the same fitting parameters as above [Fig. 10(b)] [34]. No diffraction was detected below the predicted cutoff value along $\Gamma M$ while along $\Gamma K$, diffraction was observed throughout the investigated normalized frequency range, including the bandgap between $u=0.22$ and $u=0.28$. In particular, close to the "dielectric" (valence) band edge at which diffraction efficiency approaches unity in the theory, it is measured at $90 \%$ for $u=0.21$. A simple reason for this impressive efficiency is suggested by the Ewald construction in 2-D, for a frequency of the order of the gap and an incident 
wave along $\Gamma K$ [bold arrow on Fig. 10(c)]: at the gap frequencies, two nodes of the PC's reciprocal lattice closely approach the Ewald circle, as is logical to build a Bragg reflection. But these nodes correspond to backward diffraction (thin arrows), whereas the node corresponding to specular reflection (dashed arrow) is still far from the circle and thus of lower efficiency. This naive picture neglects the various effects that relax $k$-space selection rules (finite crystal, finite wave penetration) but provides the simplest explanation of the strong backward diffraction occurring at the gap frequency.

Diffraction efficiencies in the forward geometry were also measured, but they are not displayed here, as efficiencies (predicted and measured) are all below 30\%. This pertains to the strong multiple diffraction events undergone by guided light throughout the 15 rows, which cannot, unless specifically designed, lead to a single beam. In reflection, and especially in the gap, only the penetration length of a few rows is sampled by the impinging beam, and a single diffraction event may more easily dominate.

In summary, diffraction phenomena are an integral part of photonic bandgap concepts if the outside medium is just the unetched dielectric matrix, a quite canonical case indeed: the periodic nature of photonic crystals results in the fact that light whose propagation is forbidden is not necessarily specularly reflected by the crystal. Backward diffraction is the other possible channel, which can be dominant in the bandgap region. As a consequence, photonic crystals are not, in general, perfect specular mirrors in the bandgap.

\section{Experiments with Quantum Dot Layers: Transmission}

In spite of its completeness, the above set of results is still sparse from the spectral viewpoint. In particular, we miss almost completely the "fine structure" of secondary oscillations either in transmission or reflection that are usually present in the passbands [Fig. 8(b) and (d)]. We thus investigated similar crystals, but using luminescent layers based on selforganized InAs quantum dots [39], [40]. Again, to have a full picture of the PC transmission over a broad range of the ratio $u=a / \lambda$, seven different periods were selected: $a=180,200,220,240,260,280$, and $300 \mathrm{~nm}$. For each period and orientation, PC's with a number of rows varying from $N=3$ to 15 were defined and etched with air-filling factors $f$ around 25-30\% [20].

A set taken from the "glued" data from the seven samples is presented in Fig. 11(a) for the TE- $\Gamma K$ case and $N=15$. It was treated to suppress the FP fringes by linear Fourier filtering [20]. The sharp "valence band edge" of the photonic gap is clearly defined at $u=0.21$. Apart from the leftmost data from the $a=180-\mathrm{nm}$ sample, the gap is clearly apparent, and around it, the oscillations of the fine structure are most obvious on the valence band side but are also clear in the region $u \approx 0.30$. The holes of the $a=180-\mathrm{nm}$ sample are less than $100 \mathrm{~nm}$ in diameter and limit the etch depth, causing in turn strong scattering into the substrate and weak transmission [28], [41]. The stitching mismatch between the 200- and 220-nm samples is due to the material dispersion: common spectral features (such as the valence band edge) are probed at different wavelengths for different crystal periods,

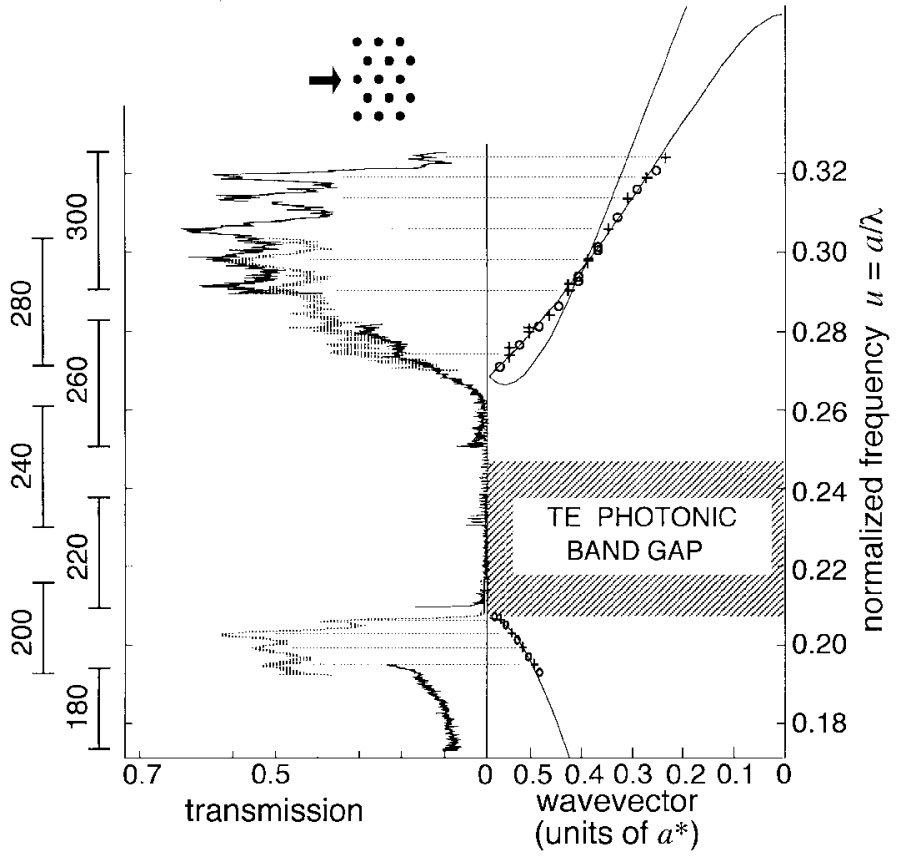

(a)

(b)

Fig. 11. (a) A set of the "glued" transmission of seven 15-row crystals of the indicated periods for TE polarization, $\Gamma K$ orientation, taken on a quantum dot-based heterostructure, as a function of the normalized frequency $u=a / \lambda$. (b) The fine structure oscillations are used to reconstruct the coupled photonic band, using $k$-quantization between the boundaries of the PC to assign the $k$ 's of oscillation extrema.

which actually correspond to crystals with different indexes of refraction. The small features into the gap correspond to the long wavelength side of the PL spectrum, with a larger noise [20].

The overall shape is in good agreement with theory [Fig. 8(b)]. The fact that the fine structure is now well seen means that Bloch waves do sample the 15 rows with a sufficiently small attenuation per round-trip to preserve these oscillations, which can be viewed as the Fabry-Perot oscillations of Bloch waves making round-trips upon reflection at the slab boundaries. The Bloch wavevector $k$ at which these oscillations occur is thus dictated by conditions similar to the classical $k$-quantization condition in a cavity of thickness $L$ ( $k L$ is a multiple of $\pi$ ). Accordingly, by plotting the frequencies of extrema at adequate $k$ 's, we could reconstruct the band structure of the crystal in the TE polarization [20], as shown in Fig. 11(b) for the coupled bands in the $\Gamma K$ direction (one of the "air" bands is symmetry forbidden) [36]-[38].

In spite of an overall agreement, there are still some interesting differences with the perfect crystal. It is worth noting, in particular, that the two bandgap edges are quite dissimilar, the "valence" band edge being much sharper than the "conduction" band edge. The reason for this difference could be intrinsic scattering losses, discussed in the next section.

\section{LIGHT LOSSES AND THEIR ORIGINS}

\section{A. Experimental Losses: The Missing Light}

As mentioned in the section on diffraction, lossless interaction of a guided wave with the structure now reads 


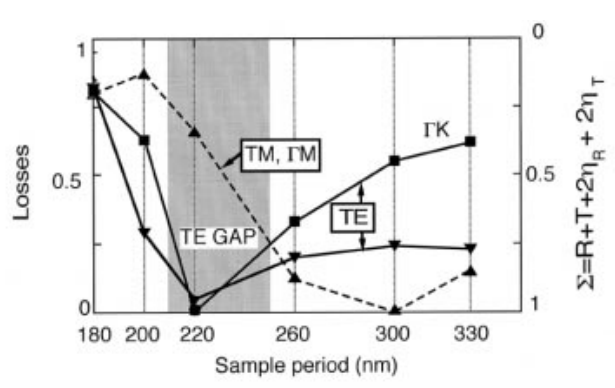

Fig. 12. Losses as a function of the sample period from the data of Figs. 8-10. Note the minimal losses in the TE gap due to high reflection or high backward diffraction efficiency and the different behavior of $T M / \Gamma M$ due to a high transmission.

$\Sigma=R+T+2 \eta_{R}+2 \eta_{T}=1$, where $D=2 \eta_{R}+2 \eta_{T}$ is the power going into diffracted waves. The out-of-plane losses are the complementary quantity $L=1-\Sigma$. The value of $\Sigma$ is specific to each sample, so we chose to present the results as a function of the lattice period rather than the variable $u$. We calculated this sum $\Sigma$ for all our quantum-well samples from $a=180$ to $330 \mathrm{~nm}$. The samples with $a=360 \mathrm{~nm}$ were not included because they exhibit second-order diffraction, which would overly complicate the analysis. The results are summarized for all samples in Fig. 12 for the TE polarization and also for the interesting case of the $\Gamma M$ direction for the TM polarization. The indicated TE gap is given for the 995-nm central wavelength of this polarization.

The high losses shown by the 180 -nm sample and probably, but to a smaller extent, by the 200-nm sample are likely to be caused by scattering arising from the insufficient etch depth due to the small hole diameters (100 and $120 \mathrm{~nm}$ ). On the other hand, the steady rise of TE losses on the "conduction band" side, contrasted by the very weak TM losses around $300 \mathrm{~nm}$ (where the crystal transmission is expected to be very close to unity even in the perfect model, as though the crystal were a stack of pseudotransparent " $\lambda / 2$ " layers), points toward losses of more intrinsic origin, i.e., intrinsic to the electromagnetic properties of the crystal + waveguide configuration, which should persist for infinite cylinder depth.

The fact that losses are minimal in the gap is good news, but it does not necessarily mean that the elementary intrinsic scattering mechanism acting in the "conduction" band is much diminished in the gap; rather, the scatterers manage to expel the electromagnetic field from the PC over a few rows by constructive interference of backward wavelets. Since the field then samples only a few rows, it experiences smaller overall losses compared to the situation where the full 15-row slab is sampled.

\section{B. The Significance of the Asymmetry of Band Edges for Losses}

The oscillations appearing in the transmission spectra of Fig. 11(a) gathered using quantum-dot emission are not as sharp as in the two-dimensional theory, and very much damped on the "air" band side. To give a heuristic explanation of

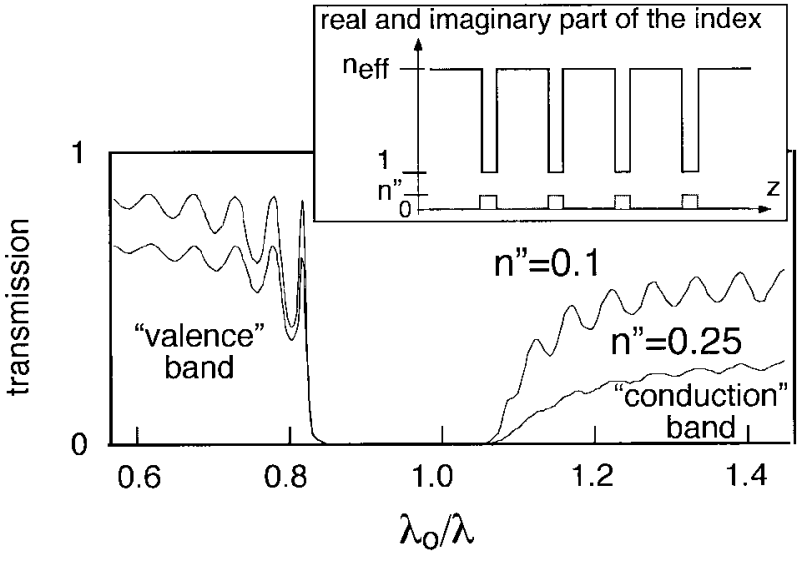

Fig. 13. Normal incidence transmission of a one-dimensional alternate periodic stack (DBR) made of lossless semiconductor $(\varepsilon=11.5)$ and a fictitious material with an index $n=1+\mathrm{in}^{\prime \prime}$, which plays the role of the photonic crystal rows of air holes, as depicted in the inset, for the two indicated values of $n^{\prime \prime}$.

the interplay of crystal modes with the loss mechanism, we present in Fig. 13 the normal incidence transmission of a one-dimensional alternate periodic stack [distributed Bragg reflector (DBR)] made of lossless semiconductor $(\varepsilon=11.5)$ and a fictitious material with an index $n=1+\mathrm{in}^{\prime \prime}$ which plays the role of the rows of air holes. Since the dissipation due to this imaginary index $n^{\prime \prime}$ arises only in air, it mimics the effect of the radiative loss mechanism, which "dissipates" in the air holes the guided waves into out-of-plane radiating modes. The filling factor of the dissipating material in this 1-D model is chosen by approximately fitting the relative gap extent of our 2-D photonic crystal.

The resulting transmissions of Fig. 13 clearly show that the lower frequency band edge is much less affected than the upper edge by the losses. It remains sharp, and secondary oscillations have about the initial amplitude for values of $n^{\prime \prime}$ such as $n^{\prime \prime}=0.2$ for which the other edge is quite smooth and display only a series of shoulders onto a smooth rising edge, with a relative amplitude growing toward higher frequency. This behavior can be simply understood from the distinct spatial localization of the two band-edge modes in a perfect stack, which are standing waves: the upper edge mode has its antinodes coinciding with the lossy material whereas, at the same location, the lower edge mode has nodes. Given the fact that the optical thickness is well below a quarter-wavelength, this latter mode becomes particularly insensitive to the losses.

The qualitative agreement between this simple picture and measurements strongly supports the conclusion that the main loss mechanism observed in our photonic crystals is indeed due to the radiation loss occurring because of the intrinsic absence of waveguiding in the air holes: the Bloch wave damping is enhanced when light is more localized in the holes, i.e., in the "air" (conduction) band. Whether the quantitative value of the observed losses corresponds to this sole physical limit (i.e., that of holes with infinite etch depth) is still an open question [42].

Another mechanism sometimes invoked is the possible poor coupling between the ordinary waveguide mode and the Bloch 
guided wave in the periodic crystal, which can have a different vertical profile. Such a mismatch might lead to out-of-plane scattering. However, as this mechanism bears little relation with the Bragg condition in the crystal, it should have no strong frequency dependence. Thus, an upper bound to its strength in terms of out-of-plane scattering is given by the point of lower losses, i.e., in the TE gap, in which it was found to be less than $10 \%$. We therefore believe that it is not our dominant loss mechanism.

\section{One-Dimensional Cavities as Probes of 2-D PC-Based Mirrors}

In the photonic gap, not only is low transmission desired, but even more desirable is high reflectivity (specular or not). While it is always difficult to calibrate reflectivities close to unity, it is obvious from the large body of work achieved in high finesse planar microcavities that measuring reflection and transmission peaks of Fabry-Perot cavities yields the most precise information regarding the mirror reflectivities [43]. An analogous horizontal cavity to the planar Fabry-Perot cavities can be created by the introduction of a spacer between two PC's, or in other words, by the introduction of a line defect in the form of a larger separation between two of the rows of a PC. The cavity is then formed by the guide section between the two mirrors [21].

This has been done for spacers in the 60-360-nm range [22], selecting the $\Gamma M$ orientation in order to get rid of diffraction effects with symmetric mirrors of four rows in each PC slab (note that the converse could have been done using $\Gamma K$ normal to the cavity and that it is possible to realize a single-mode cavity in a regime dominated by diffracted beams). The transmission characteristics of these cavities have been measured by the same method as those of PC's (the "point source" method), with some care regarding the strong angular dependence of the Fabry-Perot resonance. Sharp peaks as narrow as $8 \mathrm{~nm}$ have been measured $(Q=125)$ even for the narrowest cavities (70-nm spacer, i.e., a quarterwavelength in the guide). A typical example is shown in Fig. 14, corresponding to a "second-order" spacer (3 $\lambda / 4)$ [22] and four rows of a $200-\mathrm{nm}$ period PC. This example with a transmission at the peak of 0.2 is not the most advantageous, as peak transmission $T_{\mathrm{FP}}$ of 0.35 was also measured.

To complete the set of data, we could also measure the reflectivity of this cavity by a direct method, i.e., by locating the "point source" (the laser spot) between the PC and the cleaved edge and selecting only the reflected light, with a normalization procedure adapted to this geometry, with a $10 \%$ uncertainty. The dip in the reflectivity curve has the same narrow width as the transmission peak but goes to as low as $R_{\text {FP }}=0.25$. Still, we think that this value is overestimated and due to the divergent nature of the probe beam, among other factors that can broaden the peak.

The mirror reflectivity is relatively easy to deduce using the quality factor and the effective cavity order, which was obtained by a simple method in [22]. Finesses ranging from 25 to 63 have been deduced, translating into reflectivities in the $88-95 \%$ range for four rows. The losses can then be deduced (a)
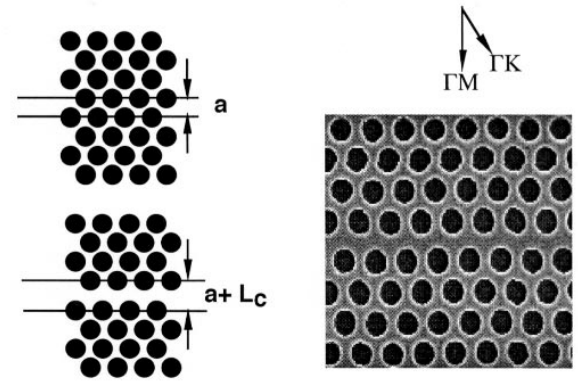

(b)

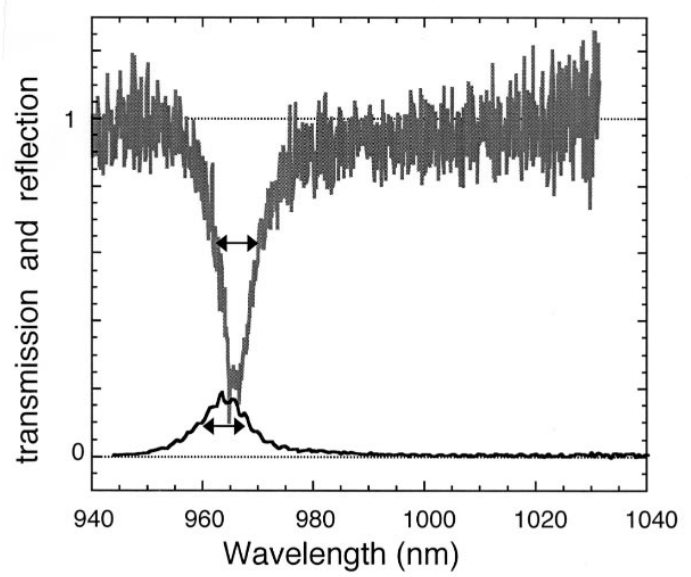

Fig. 14. (a) Schematics and micrograph of an in-plane Fabry-Perot cavity with PC mirrors (the microcavity on the micrograph has a 70-nm spacer). (b) Transmission (black) and reflection (gray) of a Fabry-Perot cavity formed between a pair of identical 2-D PC's and probed along the normal $\Gamma M$ direction. Each PC has a 200-nm period and consists of four rows. The spacer is about $200 \mathrm{~nm}$ thick.

in each mirror by calculating $1-\mathrm{R}-\mathrm{T}$, since the single mirror transmission is known to be about $4 \%$. From the above data, one can deduce losses between 2 and $8 \%$. At the present stage, our view is that the $Q$ factor of this kind of cavity gives an integrated measure of the losses but does not easily allow us to ascribe these losses to a particular location or a precise mechanism. Simple models also predict that in symmetric cavities, lossy mirrors lower the on-resonance transmission peak value much more than they affect the resonant reflection minimum, as observed in our case. Further work to understand which information can be best drawn from the combined transmission and reflection data is in progress.

\section{PHOTONiC Boxes BASED ON A WAVEGUIDE: CONFINEMENT BY CONCENTRIC BRAGG GRATINGS IN MiCRODISCS}

The ability to laterally confine light into a waveguide can be used to form fully confined photonic boxes whereby the two horizontal dimensions in the guide are subject to confinement by means of periodic etched structures while the guide freezes the third photon degree of freedom.

Such a kind of confinement was demonstrated in mushroomshaped microdiscs [44], [45] in which total internal reflection is used to confine whispering-gallery modes (WGM). Eigenmodes of a perfect disc can be indexed by an azimuthal number $m$ [the angular momentum as the field contains an $\exp (\operatorname{im} \varphi)$ 
(a)

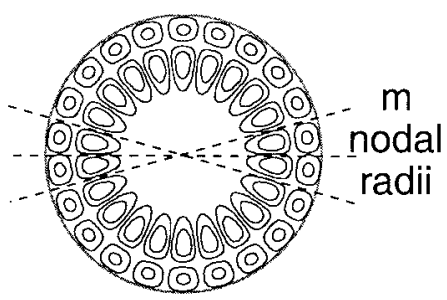

(b)

(c)

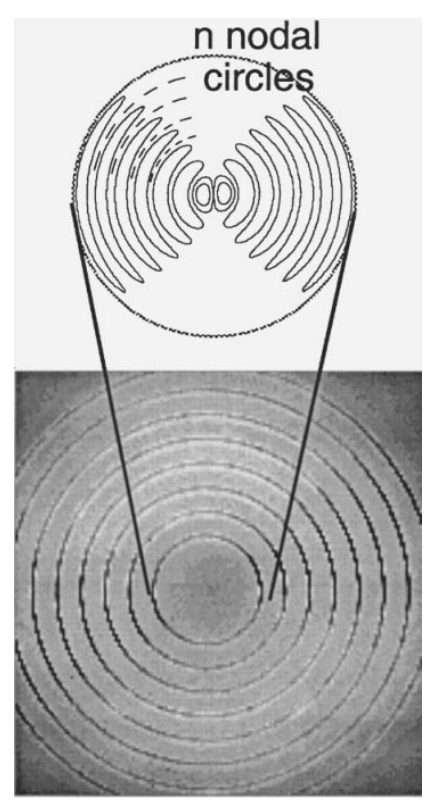

(d)

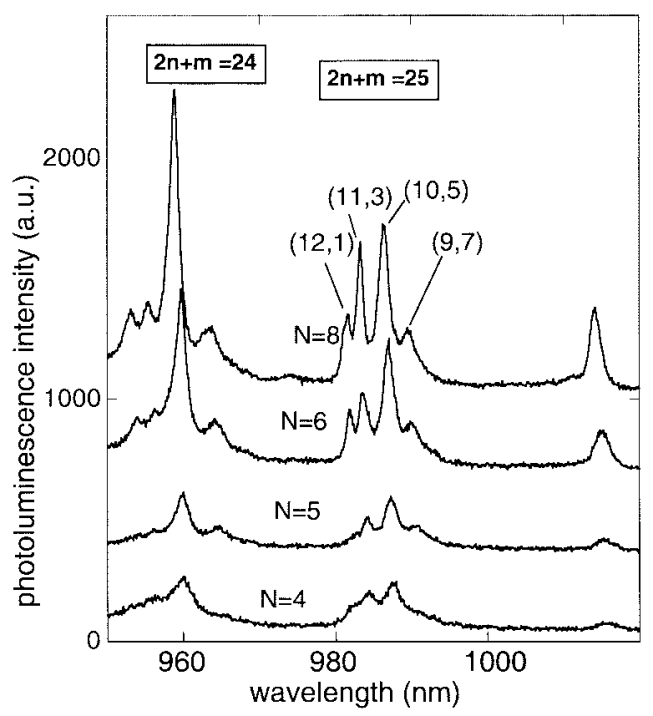

Fig. 15. (a) WGM of a perfect disc, (b) QRM's of the same disc, (c) micrograph of the disc cavity with a concentric Bragg reflector that confines QRM's, and (d) spectra of the "stray" luminescence originating from the grating area for a disc of inner diameter $2 R=3.1 \mu \mathrm{m}$, trench spacing of $\Lambda=620 \mathrm{~nm}$, and the indicated values of the number $N$ of concentric trenches. A tentative numbering of the peaks of a typical cluster with the two discrete $(n, m)$ numbers is made for the case $N=8$.

factor] and a radial number $n$. WGM's, confined close to the disc boundary, feature $m \gg n \sim 1$ and have very small modal volumes [Fig. 15(a)]. This means that, for emitters distributed across the disc, most of the emission goes into leaky modes of the disc with higher $n$ 's, not in WGM's.
We thus attempted to use a circular Bragg reflector to confine modes that do sample the entire disc, such as QRM's depicted in Fig. 15(b), with the property that $n \gg m \sim 1$ [25]. Their outer radiation pattern is more easily shaped into a useful beam [46] given their small number of lobes. A micrograph of the corresponding disc cavity is shown in Fig. 15(c). The trench spacing $\Lambda$, around $600 \mathrm{~nm}$, ensures Bragg reflection around $\lambda=1000 \mathrm{~nm}$, while the trench width was chosen small enough $(70 \mathrm{~nm})$, again to reduce radiation losses. As explained in [25], the guided light diffracted toward air at each trench is nevertheless a useful probe of guided light intensity and thus of cavity modes. The inner diameter $2 R$, around $3 \mu \mathrm{m}$, is chosen to conveniently excite into the disc the InAs dots embedded in the waveguide.

When selectively collecting the "stray" luminescence originating from the grating area, we observe the spectra of Fig. 15(d) for discs with $N=4$ to 8 trenches. As $N$ increases, these spectra display sharp clustered peaks (up to $Q \sim 900$ in some samples) onto a smooth background. These peaks could be unambiguously attributed to QRM resonances [25], each cluster corresponding to a given value of $2 n+m$, as this peculiar combination determines the QRM resonance position at first order. Details about the cluster's fine structure, governed by $\mathrm{m}^{2}$, can be found in [25] and lead to the tentative numbering shown in the figure for the case $2 n+m=25$.

Note also that the peaks do sharpen until $N=6$, but not much beyond. This value $N=6$ can be viewed as the point at which radiation losses prevent the grating reflectivity from further approaching unity: instead, the grating reflectivity saturates, as can be easily inferred again from a simple onedimensional model analogous to that of Fig. 13 above.

\section{MiCROMETER-SizEd HEXAGONAL CAVITIES BOUNDED BY 2-D PC's}

\section{A. Design and Experiments}

Defining horizontal cavities with circular boundaries allowed us to confine QRM's instead of WGM's. A still more desirable configuration is to confine all the modes of a given area by means of two-dimensionally periodic PC's that achieve an omnidirectional total reflection. Let us underline that this reflection can manifest itself in a general form including diffraction, but as long as the impinging energy flux is redirected inside the cavity, eigenmodes will form that automatically cast this diffraction phenomenon in their field pattern. For small microcavities, say, a few wavelengths, ray tracing becomes meaningless due to the diffraction limit, but one may envision larger cavities of typical size above ten wavelengths, in which a set of rays that achieve round-trips mostly based on diffraction at $\Gamma K$-type interfaces of the PC's described in Section II may be envisioned.

For simplicity with respect to simulations, and capitalizing on the microdisc ideas, we designed in-plane cavities in the form of hexagons carved into an otherwise regular triangular PC with $\Gamma M$-type boundaries, which are conveniently described by the size $h$ of their side in units of $a$ (inset of Fig. 16; similar cavities are presented in [47] and [48] and 


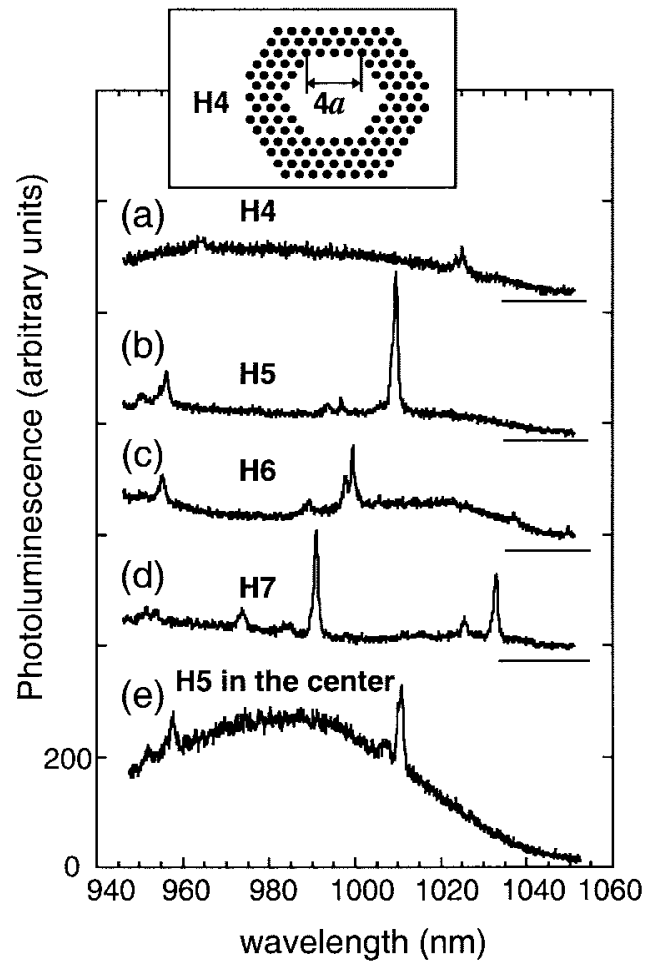

Fig. 16. Spectra for hexagonal cavities carved into triangular PC's. (a)-(d) Spectra collected in the PC area adjacent to the cavity for cavities H4-H7. The inset depicts the H4 cavity of side 4a. (e) Same cavity H5 as (b) but for collection inside the cavity, hence the larger background.

also in the contribution of Pottier et al. [49]). In our case, cavities with $h=4 a, 5 a, 6 a, 7 a$ (denoted $\mathrm{H} 4, \ldots, \mathrm{H} 7$ ) were etched in triangular photonic crystals of period $240 \mathrm{~nm}$ similar to those described in Section II. The area of the bare cavity is $S=3 \sqrt{3} h^{2} / 2$. This area is useful to estimate a lower bound to the modal volume of the modes of such a cavity as well as an upper bound to the mean mode spacing since, in two dimensions, the density of modes per unit relative bandwidth $g_{\mathrm{urb}}(\lambda)=d N /[d \lambda / \lambda]=\left(2 \pi n_{\mathrm{eff}}^{2} / \lambda^{2}\right) \times S \approx 70 S\left(\mu \mathrm{m}^{2}\right)$ around $\lambda=1 \mu \mathrm{m}$, a formula that includes possible degenerate modes that would appear experimentally as a single peak.

The basic heterostructure including an InAs QD layer (for its immunity to nonradiative recombination) and the measurement technique are the same as in Section V. The spatial resolution of light collection is now of the same order as the cavity size; also, given the broad spectrum (10\% in relative terms), chromatic aberrations blur the spatial definition. As a consequence, collection in the middle of a cavity already yields a signal that consists of a background with superimposed sharp modes. However, displacing the collection area to the PC boundary just adjacent to the bare cavity yielded a smaller background and generally larger sharp peaks. Examples of spectra collected for cavities H4-H7 are given in Fig. 16(a)-(d), while Fig. 16(e) is for H5 and a collection in the middle of the cavity, showing the large background due to the direct central area emission [23].

The interpretation of these spectra is much more difficult than for microdiscs, as there exist, to the best of our knowledge, no simple formula for the Dirichlet or Neumann eigenmodes of a hexagon, but only for those of a triangle.

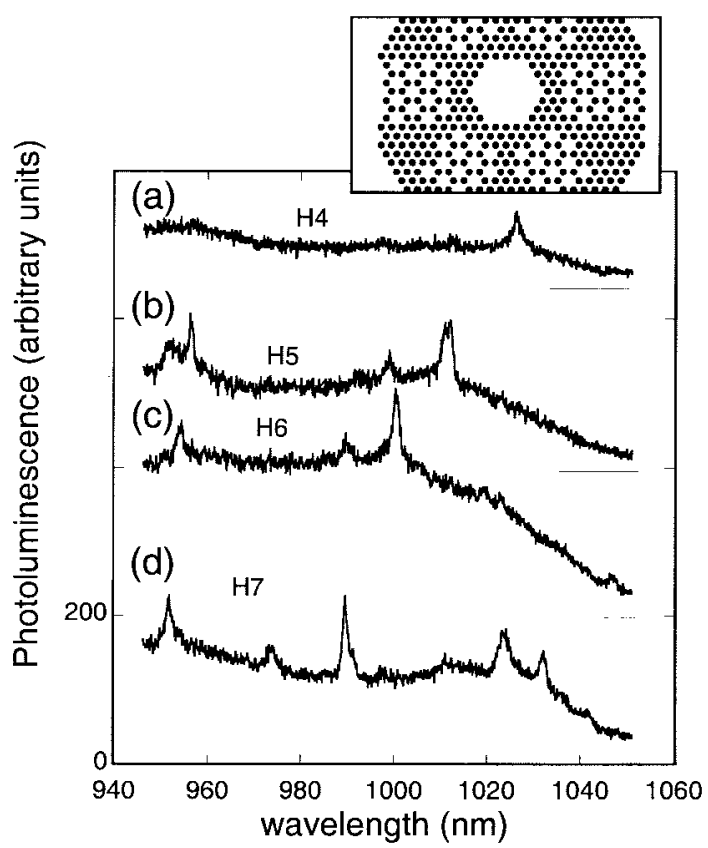

Fig. 17. Spectra for hexagonal cavities carved into modified triangular PC's. Same as Fig. 16(a)-(d) but, as depicted in the inset, sets of two or three holes parallel to the $\Gamma M$ sides are removed from the surrounding PC.

Moreover, the PC boundaries might play a role different from that of the smooth boundaries of the microdiscs by allowing different penetration lengths in different crystal directions. However, a first indication is that the number of detected peaks per unit relative bandwidth (here a 10\% relative bandwidth) is typically $1 / 4$ of the expected value in the best cavities. With the likely degeneracy effects, this discrepancy can be brought back to about one-third. A possible reason why so many of the modes are missing is probably not that they are not confined but rather that they do no scatter enough light in air to be detected, rather good news in some respects. This will appear more obviously from the mode shape given by the simulation below, with simple assumptions on the scattering mechanism.

To gain further insight on the confinement of our hexagonal cavities, we also designed similar cavities but bounded by a slightly lacunary PC, as shown in Fig. 17(a): along the $\Gamma K$ direction (from the center), a few short rows were removed. This can have various effects: degrade the quality factor, create localized or coupled resonances, and affect the air radiation mechanism in all the region "shadowed" by these missing holes. The results [Fig. 17(a)-(d)] are quite similar to the previous ones, a closer look showing that resonances now have rather lower quality factors $Q$ around 500, but at quite the same location as previously, and with very similar intensity distribution. Also, no sign of coupled or new resonances could be seen. This suggests that the light in the microhexagons is very tightly confined by the PC boundary, with a field decaying almost entirely (say one or two decades) for only three rows. Hence, the radiation mechanism responsible for the resonance detection relies essentially on the field in the two first rows. In these rows, i.e., within one wavelength, the field should be viewed as the "near field" of the cavity (rather than the "far field") following still closely the mode pattern inside the hexagon. 


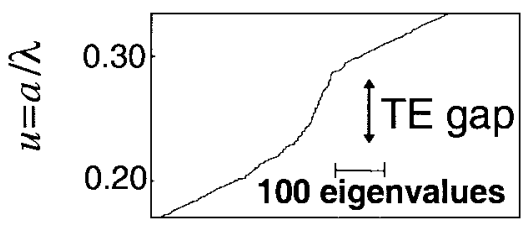

(a)

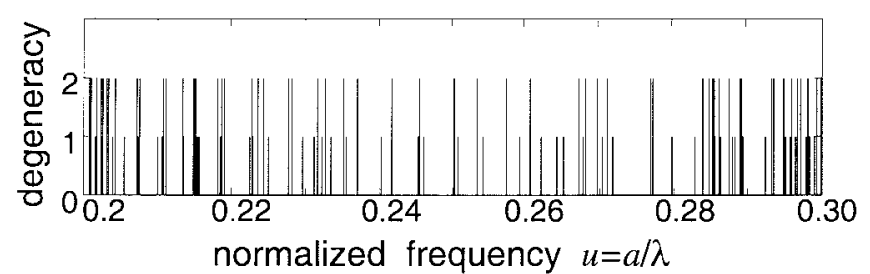

(b)

Fig. 18. Simulation of hexagonal cavity H5 (see Fig. 16). (a) Plot of the eigenvalues $\omega_{j}$ as a function of their number $j$ in which the slope is the inverse of the density-of-states, and is thus steeper when states are confined into the cavity rather than delocalized in the five times larger supercell. (b) Eigenfrequencies and their degeneracies one or two.

\section{B. Supercell Eigenmode Calculations}

These results prompted us to simulate the eigenmodes of our cavities, at least in two dimensions. We chose a plane-wave expansion on a supercell, similar to [50]. In general, one can introduce a wavevector that allow modes propagating from one supercell to the adjacent to be described. Here, we are interested only by modes localized inside a given supercell. The simplest supercell that may contain a hexagonal cavity is the lozenge of dimension $\alpha a \times \alpha a$ tiled with $\alpha^{2}$ unit cells of the triangular PC lattice. We could use sizes up to $\alpha=18$ to the expense of the accuracy since the number of reciprocal wavevectors that enter the final matrices scales like $\alpha^{2} G_{\max }$, $G_{\max }$ being the largest reciprocal wavevector, beyond which truncation occurs. In this supercell, we remove those rods that correspond to the cavity and write the structure factor for a reciprocal wavevector $\mathbf{G}$ as a sum

$$
S(\mathbf{G})=\left(\alpha^{2}\right)^{-1} \sum_{\text {rods } \mathrm{j}} \exp \left(-i \mathbf{G} \cdot \mathbf{R}_{j}\right)
$$

on the remaining rods centered at location $\mathbf{R}_{j}$. Numerically, the eigenvalue problem was solved more efficiently for a cavity centered on the supercell origin because, in this case, the sixfold symmetry results in real coefficients of $\varepsilon(\mathbf{G})$. One can think of working in each subspace associated with the eigenvalues $\exp (i n \pi / 3)$ of the sixfold rotation operator.

We first show the spectrum of the obtained cavity eigenvalues (given in terms of the normalized frequency $u=a / \lambda$ ) on Fig. 18. The inset depicts the whole eigenvalues of the matrix used in the calculation, including frequencies outside the TE gap. They are just sorted and plotted as a function of their sort number. The gap frequencies correspond to a steeper slope because, in this frequency region, all the modes are quite localized in the cavity, which is only a fraction (about 19\%) of the supercell. Hence the 2-D density-of-states of the modeled system drops from one corresponding to a large area (the supercell of area $18^{2}=324$ unit cells) to one corresponding to a smaller area (the cavity of area 61 unit cells). The difference
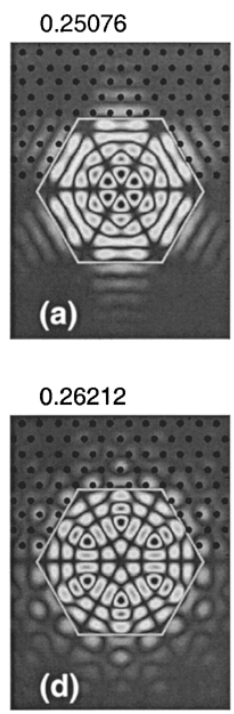
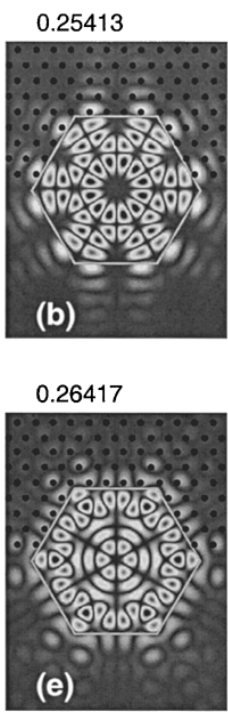

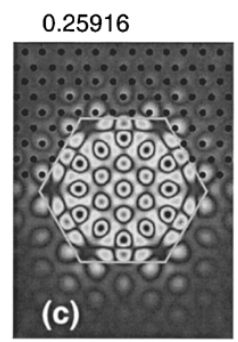

0.26518

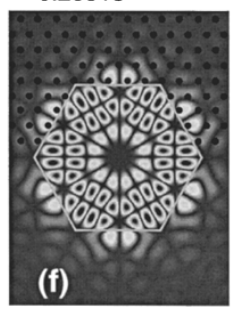

Fig. 19. Modeling of hexagonal cavities bounded by 2-D triangular PC's. (a)-(f) Typical patterns of the scalar $H$ field absolute amplitude of cavity TE modes for cavity H5 with frequencies indicated on top (into the TE photonic gap for the simulation parameters); successive nondegenerate modes were selected. The array of black circles correspond to the crystal holes. They were omitted on the bottom where only the hexagon perimeter is underlined. Note the different radiations patterns in the boundary, simple in (a), lying at corners rather than sides (b), complicated (c)-(e), or again localized on sides but with a different symmetry.

in average dielectric constant, about the $28.5 \%$ filling factor chosen in the simulation, as well as wave penetration into the PC tend to increase the density-of-states in the gap region over the naive surface-counting argument.

Typical TE mode patterns of cavity modes for cavity H5 with frequencies that fall into the proper TE photonic gap are shown in Fig. 19 through the amplitude of their scalar $H$ field. We selected nondegenerate modes for simplicity (see [23] for the pattern of a degenerate mode). A set of six such modes is illustrated in the order of increasing eigenfrequency, as indicated on top in terms of $u=a / \lambda=\omega a / 2 \pi c$. One can see that mode (a) has a single lobe along each side, mode (b) has two lobes, centered around corner, while the next modes have still more spotty patterns. A mode (f) with a rather simpler radiation pattern corresponding to a plane wave impinging at $60^{\circ}$ incidence on the side occurs again after these "spotty" modes. This selected sample gives a reasonable justification that the scattered radiation might have a very variable magnitude depending on the interference effects arising between the radiation from different part of the PC's with opposite signs on distance of the order of the crystal period. In particular, the fraction of well-detected modes might grossly coincide with the fact that only modes such as (a) and (b) give important contributions to the scattered far field in our collection geometry.

\section{CONCLUSION}

The set of measurements presented here quantitatively demonstrates in an unprecedented manner all the basic properties of two-dimensional photonic crystals in the optical range: transmission, reflection, and diffraction. It also shows that the out-of-plane losses are reasonably limited in the 
photonic gap. Another sign of this satisfactory behavior is the existence, on transmission data using a broad internal source, of the fine structure due to Bloch waves oscillating across a rather thick photonic crystal. Confinement of light by these photonic crystals was also successfully demonstrated. In-plane microcavities offer interesting performances in terms of quality factor and attractive solutions in terms of radiations patterns.

From all these features, two perspectives are opened: on the microscopic side, the choice of the heterostructure and the PC parameters can still be refined, e.g., to minimize out-of-plane scattering losses, and to tune the cutoff conditions for in-plane diffraction. The most demanding configuration for losses is probably set by the guide configuration between two PC's, which has not yet been the subject of many experiments in the near infrared.

Another challenge is to define what functions are now best fulfilled by PC's rather than by present solutions, and to what degree it should be attempted to combine into a single monolithic approach "space-consuming" integrated optics functions ( $Y$ branches, multiplexers, modulators, etc.) and their more compact PC version.

Given the progress that one can expect along these two lines, our results are very encouraging steps pointing toward the future possible uses of PC's for 2-D optoelectronic devices and circuits.

\section{REFERENCES}

[1] E. Yablonovitch, "Inhibited spontaneous emission in solid-state physics and electronics," Phys. Rev. Lett., vol. 58, pp. 2059-2062, 1987.

[2] J. D. Joannopoulos, R. D. Meade, and J. N. Winn, Photonic Crystals, Molding the Flow of Light. Princeton, NJ: Princeton Univ. Press, 1995.

[3] Y. A. Vlasov, K. Luterova, I. Pelant, B. Hönerlage, and V. N. Astratov, "Enhancement of optical gain of semiconductors embedded in three-dimensional photonic crystals," Appl. Phys. Lett., vol. 71, pp. 1616-1618, 1997

[4] S. Y. Lin, J. G. Fleming, D. L. Hetherington, B. K. Smith, R. Biswas, K. M. Ho, M. M. Sigalas, W. Zubrzycki, S. R. Kutz, and J. Bur, "A three-dimensional photonic crystal operating at infrared wavelengths," Nature, vol. 394, pp. 251-253, 1998.

[5] J. D. Fleming and S. Y. Lin, "A three-dimensional photonic crystal with stop band from 1.35 to 1.95 microns," Opt. Lett., vol. 24, pp. 49-51, 1999.

[6] S. Noda, N. Yamamoto, and A. Sasaki, "New realization method for three-dimensional photonic crystal in optical wavelength region," Jpn. J. Appl. Phys., vol. 35, pp. L909-L912, 1996.

[7] N. Yamamoto, S. Noda, and A. Cutinan, "Development of one period of three-dimensional photonic crystal in $5-10 \mu \mathrm{m}$ wavelength region by wafer fusion and laser beam diffraction pattern observation technique," Jpn. J. Appl. Phys., vol. 37, pp. 1052-1054, 1998.

[8] C. C. Cheng, A. Scherer, R.-C. Tyan, Y. Fainman, G. Witzgall, and E. Yablonovitch, "New fabrication techniques for high quality photonic crystals," J. Vac. Sci. Technol. B, vol. 15, pp. 2764-2767, 1997.

[9] Y. N. Astratov, Y. A. Vlasov, O. Z. Karimov, A. A. Kaplyanskii, Y. G. Musikhin, N. A. Bert, V. N. Bogomolov, and A. V. Prokofiev, "Photonic band gaps in 3D ordered FCC silica matrices," Phys. Lett. A, vol. 222, pp. 349-353, 1996

[10] T. F. Krauss, R. M. De La Rue, and S. Brand, "Two-dimensional photonic-bandgap structures operating at near-infrared wavelengths," Nature, vol. 383, pp. 699-702, 1996.

[11] M. D. B. Charlton, G. J. Parker, and S. W. Roberts, "Guided mode analysis, and fabrication of a two dimensional visible photonic band structure confined within a planar semiconductor waveguide," Mater. Sci. Eng. B, vol. 49, pp. 155-165, 1997.

[12] T. Baba and T. Matsuzaki, "GaInAsP/InP 2-dimensional photonic crystals," in Microcavities and Photonic Bandgaps: Physics and Applications, J. Rarity and C. Weisbuch, Eds. Dordrecht: Kluwer, 1996, vol. 324.
[13] T. Baba, "Photonic crystals and microdisk cavities based on GaInAsP-InP system," IEEE J. Quantum Electron., vol. 3, pp. 808-830, 1997.

[14] U. Grüning, V. Lehmann, S. Ottow, and K. Busch, "Macroporous silicon with a complete 2-D PBG centered at $5 \mu \mathrm{m}$," Appl. Phys. Lett., vol. 68 , pp. 747-749, 1996.

[15] H.-B. Lin, R. J. Tonucci, and A. J. Campillo, "Observation of twodimensional photonic band behavior in the visible," Appl. Phys. Lett., vol. 68, pp. 2927-2929, 1996.

[16] S.-Y. Lin, V. M. Hietala, L. Wang, and E. D. Jones, "Highly-dispersive photonic band-gap prism," Opt. Lett., vol. 21, pp. 1771-1773, 1996.

[17] A. Rosenberg, "Near infrared two-dimensional photonic band-gap materials," Opt. Lett., vol. 21, pp. 830-833, 1996.

[18] D. Labilloy, H. Benisty, C. Weisbuch, T. F. Krauss, R. Houdré, and U. Oesterle, "Use of guided spontaneous emission of a semiconductor to probe the optical properties of two-dimensional photonic crystals," Appl. Phys. Lett., vol. 71, pp. 738-740, 1997.

[19] D. Labilloy, H. Benisty, C. Weisbuch, T. F. Krauss, R. M. De La Rue, V. Bardinal, R. Houdré, U. Oesterle, D. Cassagne, and C. Jouanin, "Quantitative measurement of transmission, reflection and diffraction of two-dimensional photonic bandgap structures at near-infrared wavelengths," Phys. Rev. Lett., vol. 79, pp. 4147-4150, 1997.

[20] D. Labilloy, H. Benisty, C. Weisbuch, C. J. M. Smith, T. F. Krauss, R Houdré, and U. Oesterle, "Finely resolved transmission spectra and band structure of two-dimensional photonic crystals using InAs quantum dots emission," Phys Rev., vol. B59, pp. 1649-1652, 1999.

[21] D. Labilloy, H. Benisty, C. Weisbuch, T. F. Krauss, V. Bardinal, and U. Oesterle, "Demonstration of a cavity mode between two-dimensional photonic-crystal mirrors," Electron. Lett., vol. 33, pp. 1978-1980, 1997.

[22] C. J. M. Smith, T. F. Krauss, R. D. L. Rue, D. Labilloy, H. Benisty, C Weisbuch, U. Oesterle, and R. Houdré, "In-plane microcavity resonators with two-dimensional photonic bandgap mirrors," Inst. Elect. Eng. Proc. Optoelectron., vol. 145, pp. 337-342, 1998.

[23] _ "Near-infrared microcavities confined by two-dimensional photonic bandgap crystals," Electron. Lett., vol. 35, pp. 228-230, 1999.

[24] T. F. Krauss and R. M. De La Rue, "Exploring the two-dimension photonic bandgap in semiconductors," in Photonic Band Gap Materials, C. M. Soukoulis, Ed. Dordrecht: Kluwer, 1996, pp. 427-436.

[25] D. Labilloy, H. Benisty, C. Weisbuch, T. F. Krauss, C. J. M. Smith, R. Houdré, and U. Oesterle, "High-finesse disk microcavity based on a circular Bragg reflector," Appl. Phys. Lett., vol. 73, pp. 1314-1316, 1998.

[26] A. Mekis, S. Fan, and J. D. Joannopoulos, "Bound states in photonic crystal waveguides and waveguide bends," Phys. Rev. B, vol. 58, pp. 4809-4812, 1998.

[27] R. D. Meade, A. Deveny, J. D. Joannopoulos, O. L. Alerhand, D. A. Smith, and K. Kash, "Novel applications of photonic band gap materials: Low-loss bends and high $Q$ cavities," J. Appl. Phys., vol. 75, pp. 4753-4755, 1994.

[28] J. C. Chen, H. A. Haus, S. Fan, P. R. Villeneuve, and J. D. Joannopoulos, "Optical filters from photonic band gap air bridges," IEEE J. Lightwave Technol., vol. 14, pp. 2575-2578, 1996.

[29] J. S. Foresi, P. R. Villeneuve, J. Ferrera, E. R. Thoen, G. Steinmeyer, S Fan, J. D. Joannopoulos, L. C. Kimerling, H. I. Smith, and E. P. Ippen, "Photonic bandgap microcavities in optical waveguides," Nature, vol. 390, pp. 143-145, 1997.

[30] S. Fan, P. R. Villeneuve, J. D. Joannopoulos, and H. A. Haus, "Channel drop tunneling through localized states," Phys. Rev. Lett., vol. 80, pp. 960-963, 1996

[31] H. Benisty, H. De Neve, and C. Weisbuch, "Impact of planar microcavity effects on light extraction-I: Basic concepts and analytical trends," IEEE J. Quantum Electron., vol. 34, pp. 1612-1631, 1998.

[32] J. M. Gérard, B. Sermage, B. Gayral, B. Legrand, E. Costard, and V. Thierry-Mieg, "Enhanced spontaneous emission by quantum boxes in a monolithic optical microcavity," Phys. Rev. Lett., vol. 81, pp. 1110-1113, 1998.

[33] K. Sakoda, "Transmittance and Bragg reflectivity of two-dimensional photonic lattices," Phys. Rev. B, vol. 52, pp. 8992-9002, 1995.

[34] D. Labilloy, H. Benisty, C. Weisbuch, T. F. Krauss, D. Cassagne, C. Jouanin, R. Houdré, U. Oesterle, and V. Bardinal, "Diffraction efficiency and guided light control by two-dimensional photonic-bandgap lattices," IEEE J. Quantum Electron., to be published.

[35] M. Plihal and A. A. Maradudin, "Photonic band structure of twodimensional systems: The triangular lattice," Phys. Rev. B, vol. 44, pp. 8565-8571, 1991.

[36] W. M. Robertson, G. Arjavalingam, R. D. Meade, K. D. Brommer, A. M. Rappe, and J. D. Joannopoulos, "Measurement of photonic band structure in a two-dimensional periodic array," Phys. Rev. Lett., vol. 68, 
pp. 2023-2026, 1992.

[37] P. R. Villeneuve, S. Fan, and J. D. Joannopoulos, "Microcavities in photonic crystals: Mode symmetry, tunability, and coupling efficiency," Phys. Rev. B, vol. 54, pp. 7837-7841, 1996.

[38] K. Sakoda, "Symmetry, degeneracy, and uncoupled modes in twodimensional photonic lattices," Phys. Rev. B, vol. 52, pp. 7982-7989, 1995.

[39] J. M. Gérard, J. Y. Marzin, G. Zimmermann, A. Ponchet, O. Cabrol, D. Barrier, B. Jusserand, and B. Sermage, "InAs/GaAs quantum boxes obtained by self-organized growth: Intrinsic electronic properties and applications," Solid State Electron., vol. 40, pp. 807-814, 1996.

[40] J. M. Gérard, D. Barrier, J.-Y. Marzin, R. Kuszelewicz, L. Manin, E. Costard, V. Thierry-Mieg, and T. Rivera, "Quantum boxes as active probes for photonic microstructures: The pillar microcavity case," Appl. Phys. Lett., vol. 69, pp. 449-451, 1996.

[41] V. Berger, I. Pavel, E. Ducloux, and F. Lafon, "Finite-element Maxwell's equations modeling of etched air/dielectric Bragg mirrors," J. Appl. Phys., vol. 82, pp. 5300-5304, 1997.

[42] B. D'Urso, O. Painter, J. O'Brien, T. Tombrello, A. Yariv, and A Scherer, "Modal reflectivity in finite-depth two-dimensional photonic crystal microcavities," J. Opt. Soc. Amer. B, vol. 15, pp. 1155-1159, 1998.

[43] R. P. Stanley, R. Houdré, U. Oesterle, M. Gailhanou, and M. Ilegems, "Ultra-high finesse microcavity with distributed Bragg reflectors," Appl. Phys. Lett., vol. 65, pp. 1883-1885, 1994.

[44] S. L. McCall, A. F. J. Levi, R. E. Slusher, S. J. Pearton, and R. A. Logan, "Whispering-gallery mode microdisk lasers," Appl. Phys. Lett., vol. 60, pp. 289-291, 1992.

[45] R. K. Chang and A. J. Campillo, "Optical processes in microcavities," in Advanced Series in Applied Physics. Singapore: World Scientific, 1996, vol. 3.

[46] A. A. Tovar and G. H. Clark, "Concentric-circle-grating, surface emitting laser beam propagation in complex optical systems," J. Opt. Soc. Amer. A, vol. 14, pp. 3333-3340, 1997.

[47] R. K. Lee, O. J. Painter, B. D’Urso, A. Scherer, and A. Yariv, "Measurement of spontaneous emission from a two-dimensional photonic band gap defined microcavity at near-infrared wavelengths," Appl. Phys. Lett. vol. 71, pp. 1522-1524, 1999.

[48] A. Scherer, O. Painter, B. D'Urso, R. Lee, and A. Yariv, "InGaAsP photonic band gap crystal membrane resonators," J. Vac. Sci. Technol. $B$, vol. 16, pp. 3906-3910, 1998.

[49] P. Pottier, C. Seassal, X. Letartre, J. L. Leclercq, P. Viktorovitch, D. Cassagne, and C. Jouanin, "Triangular and hexagonal high $Q$-factor 2-D photonic bandgap cavities on III-IV suspended membranes," this issue, pp. 2058-2062.

[50] H. Benisty, "Modal analysis of optical guides with two-dimensional photonic band-gap boundaries," J. Appl. Phys., vol. 79, p. 7483, 1996.

H. Benisty, photograph and biography not available at the time of publication.
C. Weisbuch, photograph and biography not available at the time of publication.

D. Labilloy, photograph and biography not available at the time of publication.

M. Rattier, photograph and biography not available at the time of publication.

C. J. M. Smith, photograph and biography not available at the time of publication.

T. F. Krauss, photograph and biography not available at the time of publication.

Richard M. De La Rue (M'94-SM'96), for a photograph and biography, see this issue, p. 2057

R. Houdré, photograph and biography not available at the time of publication.

U. Oesterle, photograph and biography not available at the time of publication.

C. Jouanin, photograph and biography not available at the time of publication.

D. Cassagne, photograph and biography not available at the time of publication. 